\title{
A common underlying genetic contribution to the co-occurrence of depressive symptoms and lower blood pressure in adolescents
}

Yuan Ko Wang *, Jun- Ming Xu, Xin- C Yang and Huang Zhou

Department of Liver Cirrhosis, , Shanghai, China

*Corresponding Author : Yuan Ko Wang, Department of Liver Cirrhosis, , Shanghai, China ; E-mail: wangyko@126.com

Received date: October 10,2018 ;Accepted date : October 25,2018 ; Published date: November 05, 2018.

Citation : Yuan Ko Wang . A common underlying genetic contribution to the co-occurrence of depressive symptoms and lower blood pressure in adolescents, J Biomarkers and Drug Discovery. Doi: http://dx.doi.org/ 10.31579/Jbdd/2018/009.

Copyright : (c) 2018 Yuan Ko Wang. This is an open-access article distributed under the terms of The Creative Commons Attribution License, which permits unrestricted use, distribution, and reproduction in any medium, provided the original author and source are credited.

\section{Abstract}

Background: Depression and cardiovascular disease risk factors develop in childhood. The objective of this study was to investigate cross sectional and longitudinal associations between blood pressure, mood scores and tagged SNPs within the Monoamine oxidase A (MAOA) gene in the Western Australian Pregnancy Cohort (Raine) Study.

Methods: Data from the five $(n=1097)$, eight $(n=1046)$, ten $(n=1026)$ and fourteen $(n=1124)$ year surveys were used. Blood pressure was measured at all surveys, anxious-depressed scores obtained from the Childhood Behavior Checklist at all surveys and depressive symptom scores from the Beck Depression Inventory for Youth at 14 years. Single nucleotide polymorphisms (SNPs) tagging the MAOA gene were identified from HapMap Phase II (CEU) data and genotyped. Cross sectional and longitudinal analyses were used to examine the association between blood pressure (outcome) and tagged SNPs within the MAOA gene and anxious/depressed scores (outcome) and tagged SNPs within the MAOA gene.

Results: At 14 years, boys with the risk allele of SNP rs5905859 and rs3027396 had higher systolic blood pressure $\left(\beta_{\text {rs } 5905859}=2.5 ; 95 \%\right.$ Cl: $0.743,4.337$ and $\beta_{\mathrm{rs} 3027396}=2.5 ; 95 \% \mathrm{Cl}: 0.681,4.383$ respectively) and lower mood scores $\left(\beta_{\mathrm{rs} 5905859}=-0.1 ; 95 \% \mathrm{Cl}:-0.100,-0.022\right.$ and $\beta_{\mathrm{rs} 3027396}=-0.2 ; 95 \% \mathrm{Cl}:-0.313,-0.045$ respectively). Longitudinally, boys with the risk allele of SNPs rs5905859 ( $\beta=0.3 ; 95 \% \mathrm{Cl}: 0.026$, 0.540 ) or rs6609257 ( $\beta=0.3 ; 95 \% \mathrm{Cl}: 0.022,0.521$ ) had a higher mean systolic blood pressure trajectory compared to boys without.

Conclusions: Variation within or close to the MAOA gene may explain in part the association between lower depressive symptom scores and higher systolic blood pressure in Caucasian boys within the Raine cohort.

Keywords: MAOA, blood pressure, depression, children, gene.

\section{Background}

Negative mood [1] and elevated blood pressure [2] emerge in adolescent and track throughout life. In childhood, higher negative affect scores, including depressive symptoms and anxiety, have been associated with lower systolic blood pressure $[3,4]$. This association persisted after adjusting for lifestyle factors such as diet and exercise. Examining this relationship in childhood prior to the use of anti depressants and the development of common behaviourial traits, such as smoking and alcohol use, may shed some light on mechanisms underlying this association.

Numerous complex interactions between genotype, phenotype and the environment are likely to be at play [5]. This study focused on one facet, namely the hypothesis that a gene is associated with both blood pressure and anxious-depressed mood. The plausibility of this hypothesis is strengthened by heritability estimates of both blood pressure, depression and anxiety related disorders which range from $32 \%-60 \%$ [6,7], 25\%-65\% [8-11] and $48 \%[12,13]$ respectively and a study [14] that estimated blood pressure and depression share $20 \%$ of their genetic variation.

Monoamine oxidase A $(M A O A)$ gene is a potential genetic candidate that may underlie the association between negative affect and blood pressure in children. This gene was identified from a literature review using the search term "depression cardiovascular" and limited to genes implicated with both phenotypes. Each gene was individually entered into PubMed to verify that both phenotypes were in fact associated with the gene and further limited to studies published in English with 50 or more participants. Only 6 genes were identified from the initial search that were associated with depression and Cardiovascular disease risk factors, of which 1 gene MAOA, was associated separately with systolic blood pressure $[15,16]$ and depression related phenotype [17].
The MAOA gene is located on the $\mathrm{X}$ chromosome. Functionally, MAOA gene codes for an enzyme expressed in the outer mitochondrial membrane that metabolises neurotransmitters such as serotonin and norepinephrine, both of which are known to be involved in mood and in blood pressure regulation [18].

Although a search of published genome wide association studies did not yield any results for blood pressure or depression or anxiety in childhood or adulthood (http://www.genome.gov/), one study suggests the MAOA gene or nearby genes may play a role in the development of blood pressure [15] and a functional variant in the promoter region of the MAOA gene has consistently been associated with mood disorders $[17,19]$.

Previously, we found an association between low scores for depression and higher blood pressure in the 'Raine Study' West Australian children's cohort [4], the aim of the present study was therefore to see whether SNPs within MAOA were associated with both blood pressure and mood scores in this population.

\section{Methods}

\section{Sample and study design}

This study used data from the Raine Study, a prospective birth cohort of children whose mothers attended antenatal clinic at King Edward Memorial Hospital or nearby private practices in Western Australia.

Between 1989 and 1991, 2900 pregnant women were recruited at approximately 18 weeks of gestation and have previously been shown to be representative of the population presenting at King Edward Memorial Hospital [22]. Specific details of recruitment have been published elsewhere $[20,21]$. 
In this study, children were selected for analysis based on the following criteria: Singleton birth and no siblings in study, Caucasian ethnicity (both parents self-reported (82\%)), DNA sample and no congenital abnormalities.

Mood scores and certain CVD risk factors were assessed at $5,8,10$ and 14 years. The characteristics of these children have been reported elsewhere [4]. The parents of participants, as well as the participants gave informed written consent at age 14. The study was approved by Human Ethics Committees at King Edward Memorial Hospital and Princess Margaret Hospital in Perth.

\section{DNA extraction and genotyping}

A total of 10 tagged single nucleotide polymorphisms (SNPs) for MAOA gene were identified from HapMap Phase II (CEU) data using a pairwise approach in Haploview (minor allele frequency $(\mathrm{MAF})>5 \%$ and $\mathrm{r}^{2}>0.8$ ) [23]. Regions analysed include the entire gene, plus additional sequences $10 \mathrm{~kb}$ upstream and downstream of the gene.

Genomic deoxyribonucleic acid (DNA) was extracted from peripheral blood and purified according to standard protocols. MAOA SNPs were genotyped using the GoldenGate ${ }^{\circledR}$ Genotyping Assay (Illumina Inc. San Diego, California, USA) as per the manufactures protocol (Fan et al., PMID: 16847463) at the Centre for Applied Genomics (Toronto, Ontario, Canada). Briefly, SNPs were uploaded to Illumina's Assay Design Tool (ADT) (http://www.illumina.com/) for probe design as part of a custom panel (GS0010538-OPA) of 1536 SNPs. A total of $5 \mu \mathrm{l}$ of $50 \mathrm{ng} / \mu \mathrm{l}$ in 10 $\mathrm{mM}$ Tris-HCL pH 8.0, $1 \mathrm{mM}$ EDTA of genomic DNA underwent an allele specific oligonucleotide hybridization followed by extension and ligation. A universal PCR step for all 1536 loci followed with primers labelled with either Cy3 (primer 1) or Cy2 (primer 2). The amplified products were then hybridized to a sentrix array matrix (SAM) and scanned using the Illumina BeadArray Reader (BAR) (Illumina, San Diego, CA). Data were analysed with Beadstudio v.3.0 using the default parameters. Only SNPs with GenCall scores $>0.25$ were called and samples were discarded if call rates were below $85 \%$. Of the 10 tagged SNPs, all were of good quality for further analysis.

\section{Outcome measures}

Anxious-Depressed scores were available from the Child Behavior Checklist (CBCL/4-18) at all surveys [24]. The CBCL/4-18 was completed by the child's parent (usually their mother $(85 \%)$ ). The anxious-depressed subscale consisted of 13 items relating to anxious and/or depressive mood including whether the child cries a lot, is nervous, too fearful or anxious. Scores could range from zero to 26 .

Depressive symptoms were measured using the Beck Depression Inventory for Youth (BDI-Y) in the 14 year survey [25]. The BDI-Y contains 20 items relating solely to depressed symptoms that an adolescent may have felt in the last 2 weeks. Scores could range from zero to 60 . The BDI-Y was completed by the child.

Both the BDI-Y and CBCL/4-18 have excellent test-retest reliability (BDI-Y: girls: $r=0.87$; boys: $r=0.89$ ) [25] and high sensitivity (CBCL/4-18: $66 \%$ anxious-depressed) and specificity (CBCL/4- 18: 80\% anxious-depressed) [26].

Consistent with previous studies [27,28], at each survey less than $5 \%$ of the cohort was anxious-depressed as defined by Achenbach [24]. Therefore our focus was on how MAOA genotypes affected the rate of change (longitudinal) or mean measures (cross sectional) of anxious-depressed scores within the cohort [29]. For consistency we analysed the BDI-Y similarly (8.4\% were mildly, moderately or severely depressed according to the clinical thresholds defined by Beck et al., [25]).

As reported elsewhere blood pressure was measured by trained assessors [30]. Briefly, seated blood pressure was measured using a Dinamap electronic blood pressure recorder (Dinamap XL or Dinamap ProCare 100), at 2 minute intervals with the appropriate sized cuff placed on the right arm.
With the exception of blood pressure measured at year 8 when only two readings were obtained, the second and third readings recorded at 2 minute intervals were averaged for each child [31].

\section{Statistical analysis}

To identify whether MAOA SNPs were associated with mood scores and blood pressure throughout childhood two set of analysis were conducted to determine if:

1. Tagged SNPs within the MAOA gene were associated with blood pressure (outcome)

2. Tagged SNPs within the MAOA gene were associated with mood scores (outcome)

If a SNP was associated with both outcomes, blood pressure and mood measurements were included in the models above to determine that SNP was independently associated with anxious-depressed scores and systolic blood pressure respectively.

\section{Cross sectional analyses}

Cross sectional analyses were conducted using data from the 14 year survey. A multivariate linear regression model previously described [4] was used to test for associations between genotype and blood pressure in adolescents. A loge transformation was used as the continuous measure of systolic blood pressure that was not normally distributed in boys.

A mixture of generalized linear models (GLMs) (personal SAS code calling the function PROC NLMIXED) was used to account for the extra zero counts and exponential shaped distribution of BDI-Y scores and CBCL anxious-depressed scores [32]. Briefly, the model consisted of a mixture of two components, a logistic model and a negative binomial model, each of them belonging to the GLM family. The logistic model was used to estimate the probability of not reporting any mood symptoms (i.e., BDI-Y or CBCL score of zero) versus reporting at least one mood symptom (i.e., CBCL or BDI-Y scores of 1 or greater). The second part of the model assumes that the BDI-Y or CBCL scores greater than 1 followed a negative binomial distribution.

\section{Longitudinal analyses}

Longitudinal analyses were used to examine associations between MAOA genotype and anxious-depressive scores (derived from the CBCL/4-18), systolic and diastolic blood pressure. Diastolic and systolic blood pressure trajectories were constructed using linear mixed effect (LME) models [33] from 5 to 14 years. Anxious-depressed mood trajectories were constructed using a generalised linear mixed effect (GLME) model with a Poisson distribution.

All trajectories included random effects for intercept (average systolic blood pressure, diastolic blood pressure or mood score) and slope (change in systolic blood pressure, diastolic blood pressure or mood score over time). Adjustments were made for potential confounders (low income and $\mathrm{BMI})$.

Analyses were carried out separately in boys and girls due to sex specific associations between depressive symptom scores and blood pressure in this cohort [4]. Adjustments were made for the following potential confounders: age, age of first menstruation as an indicator of puberty (available for females only at 14 years), gross family income as an indicator of socio economic status, BMI and whether the adolescent smoked in the last 12 months (at 14 years only). All continuous confounders were mean centered (to the nearest whole number) to remove potential colinearity between the beta coefficients.

Significance was initially defined at the conventional 5\% level and statistical analyses were performed in the statistical package $\mathrm{R}$ version 2.6.1 [34] and SAS version 9.1 [35].

The False Discovery Rate (FDR) approach [36] using the $\mathrm{R}$ package "qvalue" was performed to correct for multiple testing The $\mathrm{q}$ value in this study corresponded to a $p$ value of $\leq 0.030$. An additive model was used unless otherwise specified. As males only have one allele (the MAOA gene is located on the $\mathrm{X}$ chromosome) Hardy-Weinberg Equilibrium was calculated for girls only. 
Post-hoc power calculations were performed using Quanto [37]. This study had $>80 \%$ power to detect genotype associations with systolic blood pressure or mood score when a SNP contributed at least $1.8 \%$ of the variation and had an allele frequency greater than $3 \%$ in girls and boys.

\section{Results}

\section{Participant characteristics and allele frequencies}

At the 5,8,10 to 14 year surveys 1097, 1046, 1026 and 1124 children, respectively, met this studies inclusion criteria. Participants that were genotyped differed only in respect to BMI when compared to those of who were not genotyped. Minor allele frequencies were greater than $3 \%$ for the study population and most were consistent with HapMap CEU frequencies. All SNPs were in Hardy-Weinberg Equilibrium.

\section{Cross sectional analysis}

\section{Genotype common to both blood pressure and mood scores}

After correcting for multiple testing, SNP rs5905859 within the MAOA gene was associated with systolic blood pressure and depressive symptom score in boys but not girls. Boys with the $\mathrm{C}$ genotype had higher systolic blood pressure and lower depressive symptom scores than boys without the $\mathrm{C}$ genotype. These associations were independent of depressive symptom score $(\beta=2.5 ; 95 \%$ CI: $0.743,4.337 ; p=0.004)$ and systolic blood pressure $(\beta=-0.1 ; 95 \% \mathrm{CI}$ : $0.100,-0.022 ; \mathrm{p}=0.002$ ) respectively. SNP rs5905859 explained $1.6 \%$ and $0.7 \%$ of the variance of systolic blood pressure and depressive symptom score (respectively). The characteristics of the study participants did not differ by rs5905859 genotype.

SNP rs3027396 was also associated with systolic blood pressure and anxious-depressed scores in boys but not girls. Boys with the A genotype had higher systolic blood pressure measurements and lower anxious-depressed scores when compared to boys without the A genotype. These associations were independent of anxious-depressed score $(\beta=2.5 ; 95 \%$ CI: $0.681,4.383 ; p=0.006)$ and systolic blood pressure $(\beta=-0.2 ; 95 \% \mathrm{CI}:-0.313,-0.045 ; \mathrm{p}=0.010)$ respectively. SNP rs3027396 explained $1.2 \%$ and $0.7 \%$ of the variance of systolic blood pressure and anxious-depressed score (respectively). The characteristics of the study participants did not differ by rs3027396 genotype.

\section{Blood pressure and genotype}

The risk alleles of the following SNPs rs6609257 or rs7052785 were associated with higher systolic blood pressure in boys. Boys with the risk alleles had $3.0 \mathrm{mmHg}$ and $6.4 \mathrm{mmHg}$ respectively higher mean systolic blood pressure compared to boys without the risk alleles.

No associations were found in girls or with diastolic blood pressure in boys.

\section{Mood scores and genotype}

Boys with the A genotype of rs 12843533 had a BDI-Y score 0.1 lower than boys without the A genotype. Girls in contrast had a BDI-Y score 0.4 higher than girls without the AA genotype.

Girls with the GG genotype of rs3027399 or GG genotype rs3027415 had a BDI-Y score 0.2 higher and 0.2 lower than girls who did not have GG genotype respectively. Boys with the A genotype of rs1181275 scored 0.2 lower on the BDI-Y than boys who did not have the A genotype.

Boys and girls with the risk allele of SNP rs3027396 and rs5906883 had anxious-depressed scores 0.1 lower than boys and girls without the risk allele.

Girls with the CC genotype of rs5905859 or GG genotype rs6609257 or rs7052785 had an anxious-depressed score $0.2,0.1$ and 0.4 lower than girls who did not have the $\mathrm{CC}$, GG or GG genotype respectively.
Boys with the $\mathrm{G}$ genotype of rs3027415 scored 0.1 higher on the CBCL anxious-depressed subscale than boys who did not have the A genotype.

\section{Longitudinal analysis}

Boys with the risk allele of rs5905859, had a greater rate of change for systolic blood pressure between 5 to 14 years (Figure 1). This persisted after adjusting for anxious-depressed score $(\beta=0.3 ; 95 \% \mathrm{CI}$ : 0.026, 0.540; $\mathrm{p}=0.031$ ).

Boys with the risk allele of rs6609257 had higher systolic and diastolic blood pressure trajectories between 5-14 years (Figure 2). Both associations persisted after adjusting for anxious-depressed score $(\beta=0.3$; 95\% CI: $0.022,0.521 ; \mathrm{p}=0.033 ; \beta=0.3 ; 95 \%$ CI: $0.053,0.449 ; \mathrm{p}=0.013$ (respectively)).No other longitudinal associations were detected.

\section{Discussion}

In a sample of Caucasian adolescents, the C genotype of SNP rs5905859 and the A genotype of SNP rs3027396 was independently associated with higher systolic blood pressure and lower mood scores in adolescent boys but not girls. Throughout childhood the risk alleles of SNPs rs6609257 and rs5905859 were associated with higher blood pressure trajectories in boys but not girls. This suggests that the MAOA gene or closely linked genes may be mutually exclusively associated with blood pressure and depression related phenotypes in Caucasian children and thus may have pleiotropic effects.

To our knowledge this is the first study to examine the pleiotro pic effects of SNPs within the MAOA gene as an explanation for the co-occurrence of depressive symptoms and blood pressure in either adults or children. The tagged SNPs we have identified within the MAOA gene, including rs5905859 and rs3027396, are located within the intronic region of the gene, do not tag the functional variant in the promoter region of the MAOA gene and to date their functional significance remains undefined (http://www.ncbi.nlm.nih.gov/projects /SNP/). The biological mechanism by which rs5905859 and rs3027396 may operate to independently affect both systolic blood pressure and mood is unknown. However these SNPs may be acting as marker for other SNPs in nearby genes that they may be in linkage disequilibrium with [38]. MAOA is part of the monoamine neurotransmitter pathway [18]. This pathway has been implicated in the degradation of neurotransmitters, such as serotonin and norepinephrine, that have been hypothesised to control mood $[39,40]$ and are known to modulate the central and peripheral autonomic nervous system that control blood pressure [41]. It is therefore plausible that the MAOA gene or nearby genes may link mood and blood pressure via the monoamine neurotransmitter pathway.

The relative small magnitude of effect and variance of SNPs rs5905859 and rs3027396 on mood scores in this study may be attributed the multifactorial nature of depression or alternatively to the low rate of "depression" within this cohort $(8.4 \%$ children could be classified as mildly, moderately or severely depressed; $>75 \%$ had a BDI-Y score less than 9 out of a possible score of 60).

The blood pressure effects are relatively large, for a population of this age and blood pressure, ranging from $2.3 \mathrm{mmHg}$ to $6.4 \mathrm{mmHg}$ for SNPs rs3027396, rs6609257 and rs7052785. The effect of these SNPs on systolic blood pressure is of potential clinical importance as relatively small changes in systolic blood pressure in adults have been shown to have significant effects on population CVD mortality rates [42]. For example a $2 \mathrm{mmHg}$ increase in systolic blood pressure in adults has been estimated to lead to a $4 \%$ increase in coronary heart disease and $6 \%$ increase in stroke [43].

There may be several explanations for the specific gender effects found. Firstly it has been suggested that sex specific associations of the MAOA gene may be due to differing patterns of monoamine metabolism and signal transduction [44]. Thus SNPs within the MAOA gene in our study may be associated with systolic blood pressure in boys but not girls because of differing hormonal environments in boys and girls, particularly during adolescence. Secondly blood pressure was substantially lower in girls than in boys with a narrower range of values so there may have been less power to show associations. 
Hypertension is more prevalent in men than premenopausal women [45] so it is possible that gender related hormonal differences may influence genetic susceptibility to systolic blood pressure. This hypothesis has been supported by a study that found systolic blood pressure to be a sexually dimorphic trait, however that study only examined genes on autosomal chromosomes [46].

Limitations of our study include lack of replication and modest statistical power. Although we have corrected for multiple testing we cannot exclude the possibility that the associations may be specific to our study population and as such replication of our findings in an independent sample is necessary to determine the external validity of our findings. The statistical power of this study was adequate to show single SNP effects but we were inadequately powered to examine gene-environment and gene-gene interactions. Given the multifactorial nature of both depression and cardiovascular disease, the hypothesis of a pleiotropic gene is only one of many possible genetic mechanisms that may contribute to both diseases.

\section{Conclusion}

Our results suggest a common underlying genetic contribution to the co-occurrence of depressive symptoms and lower blood pressure in adolescents. We have shown for the first time that lower mood scores and higher systolic blood pressure were both independently associated with SNP rs5905859 and rs3027396 in boys but not girls. In addition we have shown relatively large effects of MAOA SNPs on systolic blood pressure in boys and that MAOASNPs were associated with higher systolic blood pressure trajectories. Further studies are required to confirm our findings and examine the underlying biological mechanism as to how the MAOA gene or genes close to the MAOAgene may be contributing the co-occurrence of lower blood pressure and higher mood scores.

\section{References}

1. Costello E J, A. Erkanli and A. Angold. Is there an epidemic of child or adolescent depression? J Child Psychol Psychiatry. 2006; 47:1263-1271.

2. Chen X and Wang Y. Tracking of blood pressure from childhood to adulthood: a systematic review and meta-regression analysis. Circulation. 2008; 117:3171-80.

3. Ewart CK and Kolodner KB. Negative affect, gender and expressive style predict elevated ambulatory blood pressure in adolescents. J Pers Soc Psychol. 1994; 66:596-605.

4. Louise S, Warrington NM, McCaskie PA, Oddy WH, Zubrick SR, Hands B, Mori TA, Briollais L, Silburn S, Palmer LJ, Mattes $\mathrm{E}$ and Beilin LJ. Associations between anxious-depressed symptoms and cardiovascular risk factors in a longitudinal childhood study. Prev Med. 2012; 54:345-50.

5. Bondy B, Baghai TC, Zill P, Bottlender R, Jaeger M, Minov C, Schule C, Zwanzger P, Rupprecht R and Engel RR. Combined action of the ACE D- and the G-protein beta3 T-allele in major depression: a possible link to cardiovascular disease? Mol Psychiatry. 2002; 7:1120-6.

6. Hottenga JJ, Boomsma DI, Kupper N, Posthuma D, Snieder H, Willemsen $\mathrm{G}$ and de Geus EJ. Heritability and stability of resting blood pressure. Twin Res Hum Genet. 2005; 8:499-508.

7. Kupper N, Willemsen G, Riese H, Posthuma D, Boomsma DI and de Geus EJ. Heritability of daytime ambulatory blood pressure in an extended twin design. Hypertension. 2005;45:80-5.

8. Carmelli D, Swan GE, Kelly-Hayes M, Wolf PA, Reed T and Miller B. Longitudinal changes in the contribution of genetic and environmental influences to symptoms of depression in older male twins. Psychol Aging. 2000; 15:505-10.

9. Czajkowski N, Roysamb E, Reichborn-Kjennerud T and Tambs K. A population based family study of symptoms of anxiety and depression: the HUNT study. J Affect Disord. 2010; 125:355-60.

10. Jansson M, Gatz M, Berg S, Johansson B, Malmberg B, McClearn GE, Schalling M and Pedersen NL. Gender differences in heritability of depressive symptoms in the elderly. Psychol Med. 2004; 34:471-9.
11. Orstavik RE, Kendler KS, Czajkowski N, Tambs K and ReichbornKjennerud T. Genetic and environmental contributions to depressive personality disorder in a population-based sample of Norwegian twins. J Affect Disord. 2007; 99:181-9.

12. Kendler KS, Karkowski LM and Prescott CA. Fears and phobias: reliability and heritability. Psychol Med. 1999; 29:539-53.

13. Stein MB, Jang KL and Livesley WJ. Heritability of social anxietyrelated concerns and personality characteristics: a twin study. J Nerv Ment Dis. 2002; 190:219-24.

14. Scherrer JF, Xian H, Bucholz KK, Eisen SA, Lyons MJ, Goldberg J, Tsuang $\mathrm{M}$ and True WR. A twin study of depression symptoms, hypertension, and heart disease in middle-aged men. Psychosom Med. 2003; 65:548-57.

15. Harrap SB, Wong ZY, Stebbing M, Lamantia A and Bahlo M. Blood pressure QTLs identified by genome-wide linkage analysis and dependence on associated phenotypes. Physiol Genomics. 2002; 8:99-105.

16. Robertson D. Genetics and molecular biology of hypotension. Curr Opin Nephrol Hypertens. 1994; 3:13-24.

17. Fan M, Liu B, Jiang T, Jiang X, Zhao H and Zhang J. Meta-analysis of the association between the monoamine oxidase-A gene and mood disorders. Psychiatr Genet. 2010; 20:1-7.

18. Brunner HG, Nelen M, Breakefield XO, Ropers HH and van Oost BA. Abnormal behavior associated with a point mutation in the structural gene for monoamine oxidase A. Science. 1993; 262:57880 .

19. Schulze TG, Muller DJ, Krauss H, Scherk H, Ohlraun S, Syagailo YV, Windemuth C, Neidt H, Grassle M, Papassotiropoulos A, Heun R, Nothen MM, Maier W, Lesch KP and Rietschel M. Association between a functional polymorphism in the monoamine oxidase A gene promoter and major depressive disorder. Am $J$ Med Genet. 2000; 96:801-3.

20. Newnham JP, Doherty DA, Kendall GE, Zubrick SR, Landau LL and Stanley FJ. Effects of repeated prenatal ultrasound examinations on childhood outcome up to 8 years of age: follow-up of a randomised controlled trial. Lancet. 2004; 364:2038-44.

21. Newnham JP, Evans SF, Michael CA, Stanley FJ and Landau LI. Effects of frequent ultrasound during pregnancy: a randomised controlled trial. Lancet. 1993; 342:887-91.

22. Evans S, Newnham J, MacDonald W and Hall C. Characterisation of the possible effect on birthweight following frequent prenatal ultrasound examinations. Early Hum Dev. 1996; 45:203-14.

23. Barrett JC, Fry B, Maller J and Daly MJ. Haploview: analysis and visualization of LD and haplotype maps. Bioinformatics 2005; 21:263-5.

24. Achenbach T.M. Manualfor the Child Behavior Checklist/4-18 and 1991 Profile. Burlington: University of Vermont, Department of Psychiatry. 1991.

25. Beck J S, A T Beck and J B Jolly. Beck Youth Inventories of Emotional and Social Impairment. United States of America: The Psychological Corporation. 2001.

26. Zubrick S.R et al. Western Australian Child Health Survey: Education, Health and Competence. Australian Bureau of Statistics and the TVW Telethon Institute for Child Health Research: Perth, Western Australia. 1997.

27. Costello EJ, Foley DL and Angold A. 10-year research update review: the epidemiology of child and adolescent psychiatric disorders: II. Developmental epidemiology. J Am Acad Child Adolesc Psychiatry. 2006; 45:8-25.

28. Sawyer MG, Arney FM, Baghurst PA, Clark JJ, Graetz BW, Kosky RJ, Nurcombe B, Patton GC, Prior MR, Raphael B, Rey JM, Whaites LC and Zubrick SR. The mental health of young people in Australia: key findings from the child and adolescent component of the national survey of mental health and well-being. Aust $N \quad Z \quad J$ Psychiatry. 2001; 35:806-14.

29. Pine DS, Cohen P, Brook J and Coplan JD. Psychiatric symptoms in adolescence as predictors of obesity in early adulthood: a longitudinal study. Am J Public Health. 1997; 87:1303-10. 
30. Huang RC, Mori TA, Burke V, Newnham J, Stanley FJ, Landau LI, Kendall GE, Oddy WH and Beilin LJ. Synergy between adiposity, insulin resistance, metabolic risk factors, and inflammation in adolescents. Diabetes Care. 2009; 32:695-701.

31. Schulze MB, Kroke A, Bergmann MM and Boeing $\mathrm{H}$. Differences of blood pressure estimates between consecutive measurements on one occasion: implications for inter-study comparability of epidemiologic studies. Eur $J$ Epidemiol. 2000; 16:891-8.

32. Tooze JA, Grunwald GK and Jones RH. Analysis of repeated measures data with clumping at zero. Stat Methods Med Res. 2002; 11:341-55.

33. Laird NM and Ware JH. Random-effects models for longitudinal data. Biometrics. 1982; 38:963-74.

34. R Development Core Team R. A language and environment for statistical computing. R Foundation for Statistical Computing: Vienna, Austria. 2007.

35. SAS Institute Inc., SAS. 2002-2003: Cary, NC, USA.

36. Storey J D. A direct approach to false discovery rates. Journal of the Royal Statistical Society. 2002; 64:479-498.

37. Gauderman WJ. Sample size requirements for association studies of gene-gene interaction. Am J Epidemiol. 2002; 155:478-84.

38. Goldstein DB and Weale ME. Population genomics: linkage disequilibrium holds the key. Curr Biol. 2001; 11:R576-9.
39. Castren E. Is mood chemistry? Nat Rev Neurosci. 2005; 6:241-6.

40. Lucki I. The spectrum of behaviors influenced by serotonin. Biol Psychiatry. 1998; 44:151-62.

41. Haavik J, Blau N and Thony B. Mutations in human monoaminerelated neurotransmitter pathway genes. Hum Mutat. 2008; 29:891902.

42. Rose G. Strategy of prevention: lessons from cardiovascular disease. Br Med J (Clin Res Ed). 1981; 282:1847-51.

43. Stamler J, Rose G, Stamler R, Elliott P, Dyer A and Marmot M. INTERSALT study findings. Public health and medical care implications. Hypertension. 1989; 14:570-7.

44. Karayiorgou M, Sobin C, Blundell ML, Galke BL, Malinova L, Goldberg P, Ott J and Gogos JA. Family-based association studies support a sexually dimorphic effect of COMT and MAOA on genetic susceptibility to obsessive-compulsive disorder. Biol Psychiatry. 1999; 45:1178-89.

45. Wiinberg N, Hoegholm A, Christensen HR, Bang LE, Mikkelsen KL, Nielsen PE, Svendsen TL, Kampmann JP, Madsen NH and Bentzon MW. 24-h ambulatory blood pressure in 352 normal Danish subjects, related to age and gender. Am J Hypertens. 1995; 8:978-86.

46. Weiss LA, Pan L, Abney $M$ and Ober C. The sex-specific genetic architecture of quantitative traits in humans. Nat Genet. 2006; 38:218-22. 